\title{
Identifying Method of Drunk Driving Based on Driving Behavior
}

\author{
Xiaohua Zhao*, Xingjian Zhang, Jian Rong \\ Key Lab of Traffic Engineering, Beijing University of Technology, \\ Beijing, 100124, P.R.China \\ Jianming Ma \\ Traffic Operations Division, Texas Department of Transportation, \\ Austin, TX 78701-2483, the university states, \\ Email: jianming.ma@txdot.gov, \\ http://www.txdot.gov \\ Received: 08-03-2011 \\ Accepted: 20-04-2011
}

\begin{abstract}
Drunk driving is one of the leading causes contributing to traffic crashes. There are numerous issues that need to be resolved with the current method of identifying drunk driving. Driving behavior, with the characteristic of real-time, was extensively researched to identify impaired driving behaviors. In this paper, the drives with BACs above $0.05 \%$ were defined as drunk driving state. A detailed comparison was made between normal driving and drunk driving. The experiment in driving simulator was designed to collect the driving performance data of the groups. According to the characteristics analysis for the effect of alcohol on driving performance, seven significant indicators were extracted and the drunk driving was identified by the Fisher Discriminant Method. The discriminant function demonstrated a high accuracy of classification. The optimal critical score to differentiate normal from drinking state was found to be 0 . The evaluation result verifies the accuracy of classification method.
\end{abstract}

Keywords: driving behavior, drunk driving, identifying indicators, Fisher discriminant function.

\section{Introduction}

Roadway traffic safety has been a popular topic over the last several decades in China. As the number of vehicles and vehicle miles traveled increase, numerous challenges facing traffic safety researchers, practitioners and policymakers have arisen. It is indicated that nearly $80 \%$ of traffic crashes are caused by drivers in the road traffic system $^{1-3}$. As one of the main causes of severe traffic crashes, drunk driving is especially prominent. According to a survey conducted by the World Health Organization (WHO), $50 \% \sim 60 \%$ of traffic crashes are related to drunk driving, and the proportion of casualties reaches $25 \%{ }^{4}$. Even with a small amount of alcohol assumption, drivers are twice likely to be involved in traffic crashes than sober drivers ${ }^{5-6}$.

Many countries have been working on solutions to drunk driving for a long period, including physiological testing, and tough drunk-driving laws. But there are a few issues regarding timeliness, accuracy and feasibility of existing

*Corresponding author: zhaoxiaohua@bjut.edu.cn 
physiological testing methods. These issues make it difficult to efficiently enforce drunk-driving laws. In fact, as we know that driving behavior is related directly to traffic crashes. Previous studies result ${ }^{6-9}$ suggests that drinking affects drivers' driving behavior and reduces their ability to control the vehicle.

For this reason, a new driving behavior based method to identify drunk driving was proposed in this paper. Firstly, according to the definition of the two driving states, normal and drunk driving, a driving simulator was used to collect driving behavior data of the two driving states. Additionally, by analyzing the difference of driving behavior under the influence of alcohol, how to identify drunk driving based on driving behavior is explored. Finally, a comprehensive indicator of driving behavior was developed to help identify drunk driving.

\section{The Experiment Design and Data Collection}

Considering its particularity, driving simulator is often used to research the dangerous driving behaviors such as drunk driving. SIMWORD, a driving simulator, was applied to capture driving behavior data including vehicle movement and maneuver operation.

\subsection{Experimental Scenario}

All road environments of the experimental scenario were designed in accordance with the road design criteria to improve the authenticity. The scenario was designed to make drivers perform, starting, accelerating, decelerating, stopping, maintaining speed and turning, 6 maneuvers. Fig. 1 shows each maneuver in the experimental scenario.

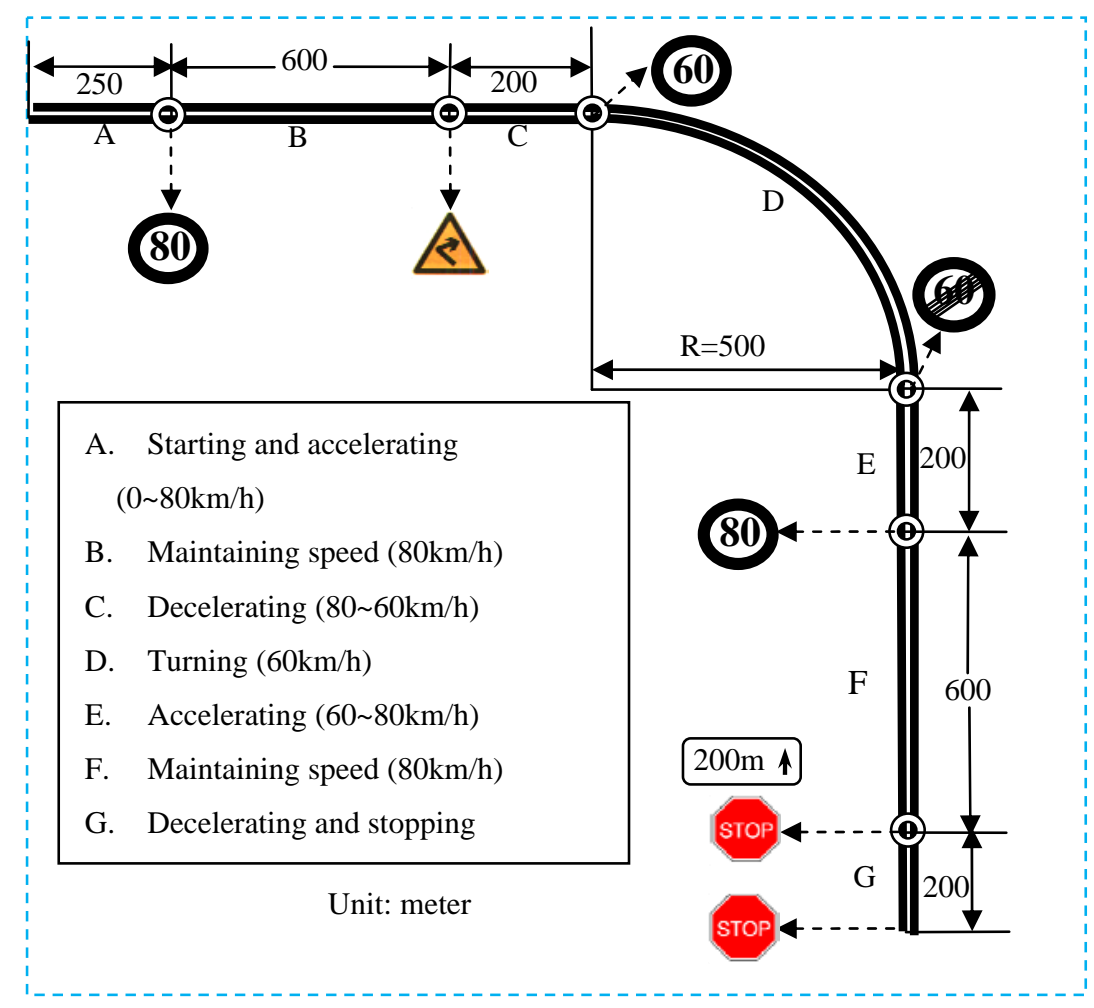

Fig. 1 Experimental Scenario Design

\subsection{Subjects}

According to a survey by Fudan University ${ }^{10}$, male drivers of 20 to 40 years of age experience the highest crash rate when driving under intoxication, accounting for $50 \%$ of all drunk-driving related crashes. Therefore,
24 male drivers between 20 to 40 years old were recruited, and these drivers had at least 3 years of regular driving experience. Blood Alcohol Concentration (BAC) is a most common metric of intoxication for legal purposes. According to a 2004 report by World Health Organization $(\mathrm{WHO})^{11}$, the risk of traffic crashes when 
the drivers' $\mathrm{BAC}$ reaches $0.05 \%$ is twice as that when the BAC is 0 . Therefore, drivers with $0.05 \%$ BAC are defined as drunk driving in the study.

\subsection{Experimental Process}

The main purpose of this experiment is to obtain the behavioral data of normal and drunk driving. In order to avoid interferences from other factors such as fatigue, the experiment was conducted at the same time on a two-day period. All of the experiments were done at 14:00 - 16:00 and the drivers' order and driving-time were kept the same. Each driver respectively drove two trips, normal and drunk states. And the questionnaire was filled out before each test to ensure the same initial states. The drunk-driving experiment was carried out when the drivers' BAC reached and exceeded approximately $0.05 \%$. The experimental process is shown in Fig.2. The data detected in driving simulator included throttle, brake and steering as well as the vehicle speed and acceleration.

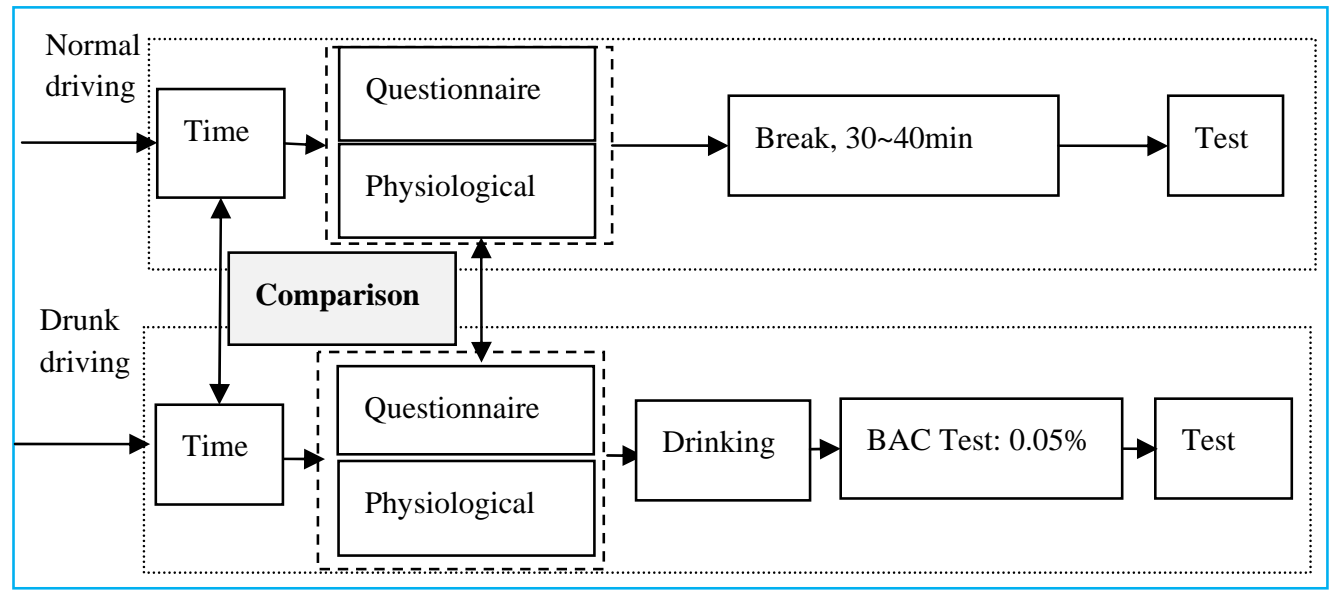

Figure 2: The Experimental Process Design

\section{Data Analysis}

The driver's behavior data of 17 drivers randomly selected of 24 participants was analyzed. The rest will be used as the availability evaluation.

\subsection{Defining of Characteristic Parameters}

Alcohol can affect drivers' central nervous system, make the ability of perception and cognition decline, and influence the drivers' emotions and increase their crash risk $^{12-13}$. It also can increase reaction time and weaken the judging ability and visual-motor coordination ${ }^{14}$. The braking response time is about 2.2 seconds longer than the average. As said in the Weiler report ${ }^{7}$, the alcohol affects the drivers' ability of maintaining traffic lanes. The previous research indicated drinking will make drivers be excited, over-confident and lead to speeding ${ }^{8}$. In the process of driving, the influence of alcohol will be shown in two aspects of running situation of vehicles and operation behavior of the drivers ${ }^{15}$. The ability of controlling vehicles is expressed by the control of speed, acceleration, and travel path of the vehicles. Operation behavior mainly includes various pressing and releasing (gas, clutch, or brake pedals) operations, and steering control. The definitions of the characteristic parameters are given in Table 1. Based on the impacting mechanism of alcohol on the drivers' psychological status and their ability of judgment and perception, we select the candidate indicators and intend to narrow down to one or two specific driving behavior parameters, which can help differentiate drunk driving from normal driving.

\subsection{Effects of Drunk Driving on Drive behavior}

Alcohol impairs drivers' performance ability. The key task of this section is to study the change of driving behavior under the influence of alcohol. It will be the foundation for identifying drunk driving. Two aspects of vehicle running state and operating performance were researched. 
Table 1 Definition of Characteristic Parameters

\begin{tabular}{|c|c|c|c|}
\hline Aspect & Object & Characteristic Parameters & Numerical Methods of parameters \\
\hline \multirow{4}{*}{$\begin{array}{l}\text { Vehicles } \\
\text { Running }\end{array}$} & Speed & Running Speed & Average speed in maintaining speed section \\
\hline & Acceleration & Average Acceleration & Average acceleration value in accelerating process \\
\hline & Deceleration & Average Deceleration & Average acceleration value in decelerating process \\
\hline & Running path & Offset to Centerline & Standard deviation (S.D) of the offset to centerline when turning \\
\hline \multirow{10}{*}{$\begin{array}{l}\text { Operating } \\
\text { Behavior }\end{array}$} & \multirow[b]{2}{*}{ Clutch } & Releasing speed when starting & (Depth after releasing - depth before releasing)/time used for starting \\
\hline & & $\begin{array}{l}\text { Releasing speed when } \\
\text { shifting gears }\end{array}$ & $\begin{array}{l}\text { (Depth after releasing - depth before Releasing)/time used for shifting } \\
\text { gears }\end{array}$ \\
\hline & \multirow{3}{*}{ Brake } & Brake Times & Number of brake operations to complete a specific task \\
\hline & & Brake Speed & (Depth before Braking - depth after braking)/time used for braking \\
\hline & & Brake Depth & The maximum depth of brake pedal to finish a specific ask \\
\hline & \multirow{3}{*}{ Gas Pedal } & Initial Speed of Pedaling & (Depth before pedaling - depth after pedaling)/time used for starting \\
\hline & & $\begin{array}{l}\text { Pedaling Speed when } \\
\text { Accelerating }\end{array}$ & $\begin{array}{l}\text { (Depth before pedaling - depth after pedaling)/time used for } \\
\text { accelerating }\end{array}$ \\
\hline & & Pedaling Depth & $\begin{array}{l}\text { The maximum depth of pedaling on the gas pedal to finish a specific } \\
\text { task }\end{array}$ \\
\hline & $\begin{array}{l}\text { Reaction } \\
\text { Time Interval }\end{array}$ & Time Interval & Time from releasing throttle to next pushing brake \\
\hline & $\begin{array}{l}\text { Steering } \\
\text { Wheel }\end{array}$ & Turning Complexity & S.D of the turning angle of the steering wheel \\
\hline
\end{tabular}

\subsubsection{Effects on Vehicle Running State}

As the combination of vehicle speed and running direction, the vehicle running state is the core of the driver control. According to the Table 1, the vehicle speeds and running path were analyzed in this study.

Vehicle speeds reflect driver's mentality, perception and operation ability. The stability of speed will be declined under the influence of alcohol. Fig.3 shows the comparison of characteristic parameters of vehicle running state under the condition of normal and drunk driving. In Fig.3, (a) and (b) are the typical comparison of the speed change from accelerating to constant speed, and then decelerating processes. The two figures indicate that when the driver was in drunk driving state, the values of acceleration, deceleration and speed will be higher, and the operation of decelerating will be delayed. This finding may be due to the fact that drivers will be excited, excessively self-confident and the judgment ability is impaired under the influence of alcohol.

The detailed analysis of the 17 drivers' vehicle running states data included the mean speed on the maintaining speed segment (B and F of Fig.1), the mean acceleration on the accelerating segment (A and $\mathrm{E}$ of Fig.1), and the mean deceleration on the decelerating segment $(\mathrm{C}$ and $\mathrm{G}$ of Fig.1). (c) To (e) of Fig.3 show the comparisons of normal and drunk driving. The results further suggest that drivers take a higher risk after drinking.

The significance analyses of the above characteristic parameters are shown in Table 2. The analysis results prove that the characteristic parameters of vehicle running state, including the speed, acceleration and deceleration, are all significantly influenced by alcohol.

*Corresponding author: zhaoxiaohua@bjut.edu.cn 


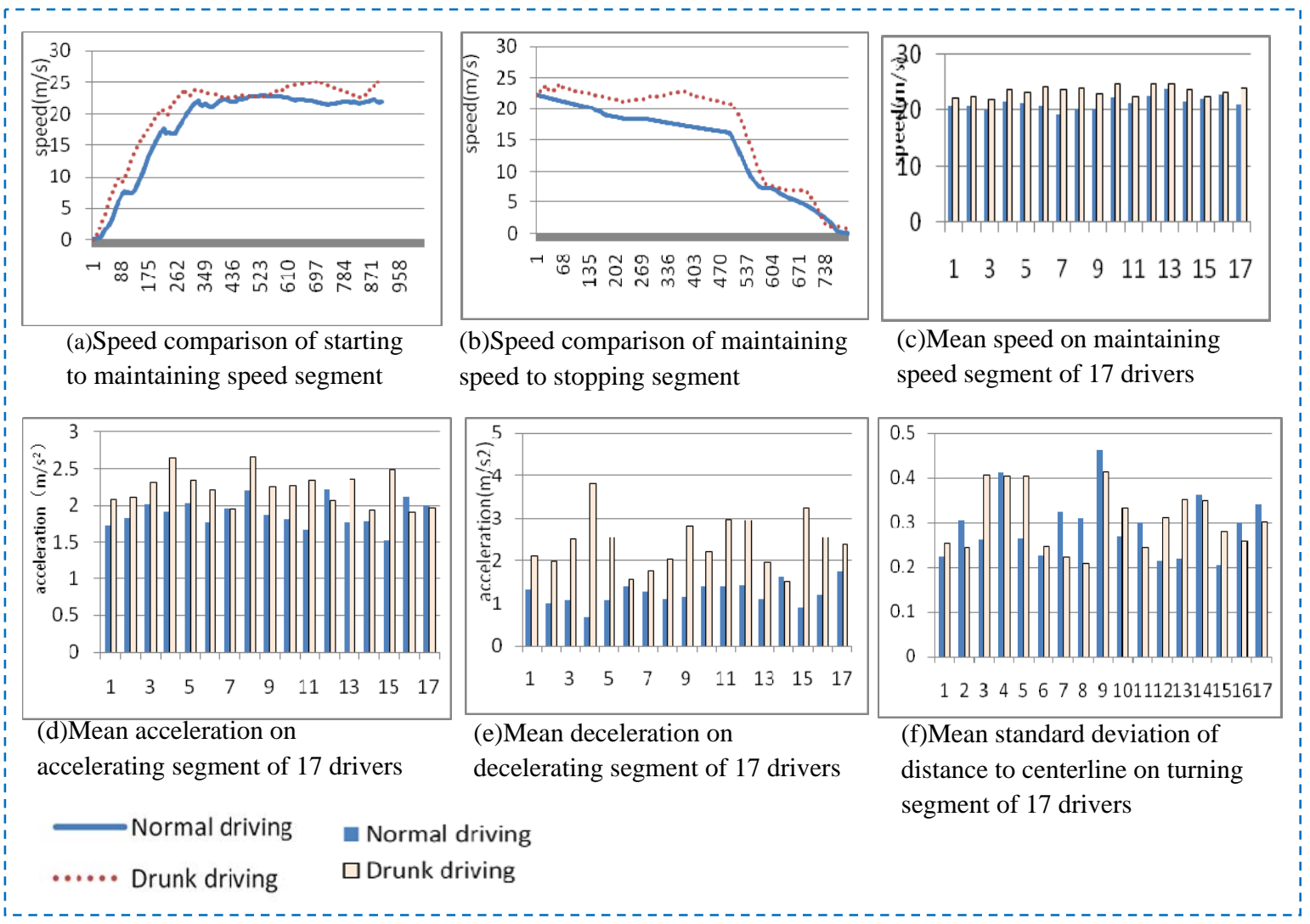

Fig. 3: The comparison of characteristic parameters of vehicle running state

Table 2 The significance Analyses of the Characteristic Parameters of Vehicle Running State

\begin{tabular}{|c|c|c|c|c|c|}
\hline \multirow{2}{*}{ Parameter } & \multicolumn{2}{|c|}{ Mean } & \multicolumn{2}{c|}{ Standard deviation } & P value \\
\cline { 2 - 6 } & Normal driving & Drunk driving & Normal driving & Drunk driving & \\
\hline Mean speed & 21.1749 & 23.2655 & 1.1041 & 0.9033 & 0 \\
\hline Acceleration & 1.8946 & 2.2306 & 0.1866 & 0.2314 & 0 \\
\hline Deceleration & 1.2289 & 2.4063 & 0.2641 & 0.6168 & 0 \\
\hline $\begin{array}{c}\text { Mean S.D of offset } \\
\text { to the centerline }\end{array}$ & 0.2939 & 0.3075 & 0.0697 & 0.0679 & 0.505 \\
\hline
\end{tabular}

\subsubsection{Effects on Operation Performance}

Drivers control a car starting, accelerating, maintaining speed, decelerating, stopping and turning mainly through the clutch, gas pedal, brake, steering wheel, hand brake and other control devices in the vehicle.

Fig. 4 shows the mean speed of releasing the clutch in process of starting vehicle and shifting gears. It can be shown that the speed of releasing clutch for drunk drivers is higher than that for normal drivers. For throttle control, we analyzed mainly the depth and speed of pedaling, as shown in Fig.5. The reason to divide the pedaling speed into two aspects is that the situation of pedaling throttle when starting vehicle and accelerating is different. Fig.5 shows that both the pedaling depth and speed are changed under the influence of alcohol, and deeper and higher. The changes of both clutch and throttle may lie in the same reasons that tactile perception of drunk drivers is 
dropped as they are over-confident, and thus drivers and speeding up. under influence of alcohol are more likely getting excited

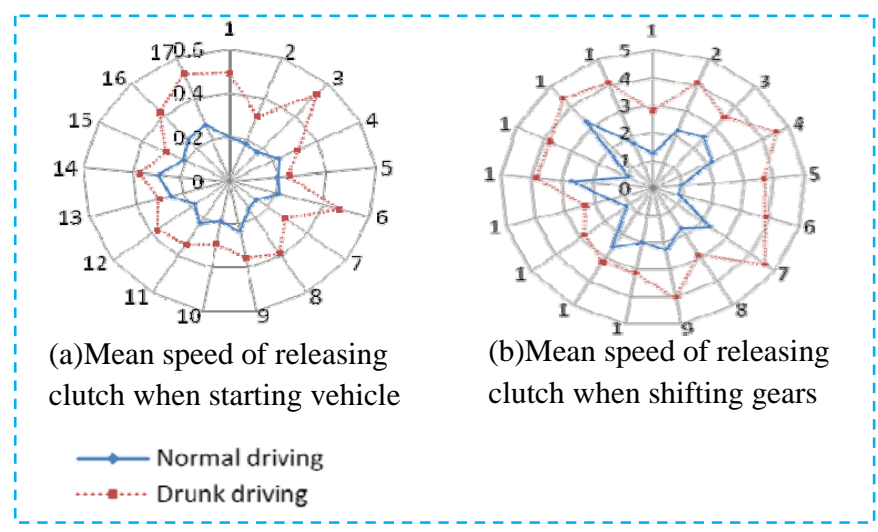

Fig.4 Comparison of Characteristic Parameters of Clutch

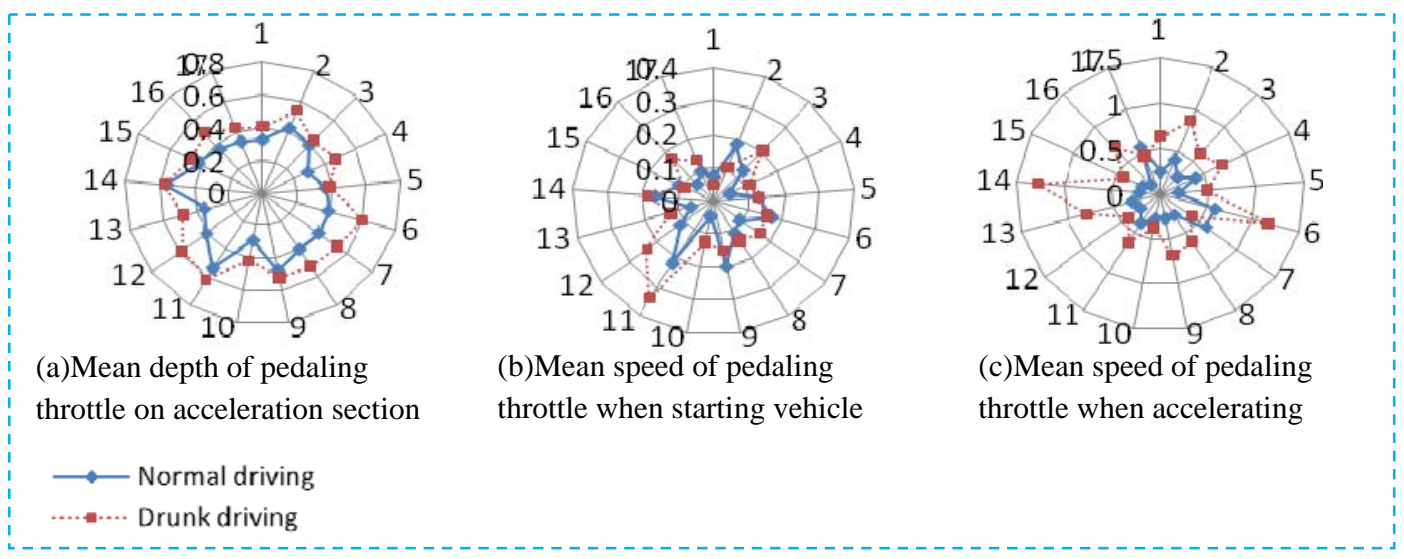

Fig. 5 Comparison of Characteristic Parameters of Gas Pedal

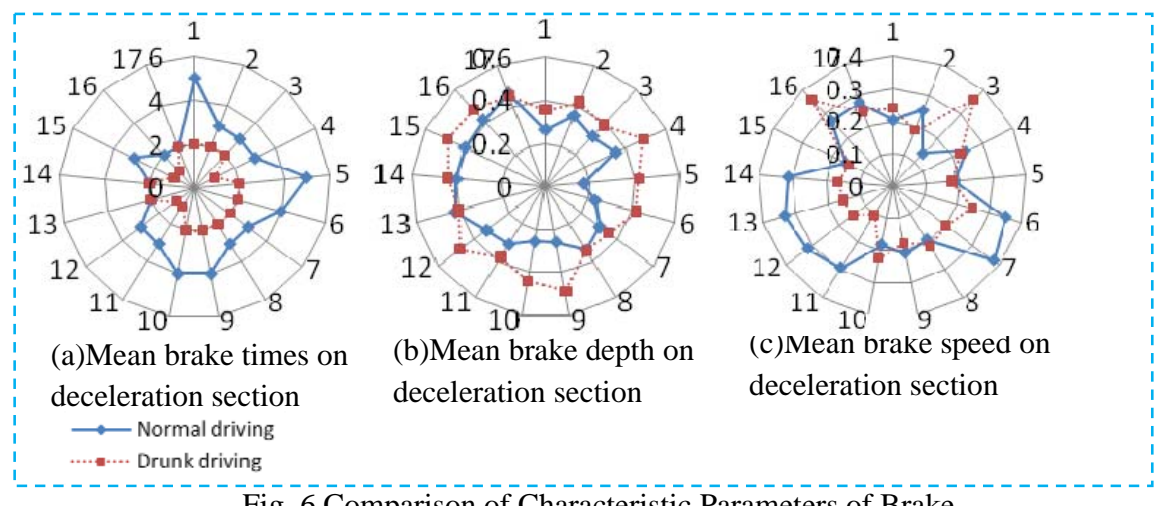

Fig. 6 Comparison of Characteristic Parameters of Brake 
Fig.6 shows the differences of braking operations between normal and drunk driving. It can be seen from Fig.6 (a) and (b) that the brake times decreases and the brake depth increases in drunk driving. But there are no remarkable changes in brake speeds according to Fig.6(c). This is due to the same reason that drinking makes drivers over-confident and their visual and judging ability is impaired.

Research shows that alcohol seriously affects drivers' reaction time, which is one of the main reason contributing to traffic crashes. The driver, judging the road traffic environment, releases the throttle firstly and then steps on the brake to slow down when driving. This paper defined the time interval between the two kinds of operations of throttle and brake as an indicator of driver reaction time interval. Fig.7 (a) shows the mean time interval between releasing throttle and braking. When normal driving, drivers had timely judgment about road environment and would release the throttle in advance when needing to brake, there was a longer interval between the throttle and braking. But in the same situation when drunk driving, drivers' reaction speed slowed down and couldn't judge traffic environment in time. So when it was needed braked, drivers would

release the throttle later and then brake immediately, with a shorter time interval.

Figure 7(b) indicates that the steering wheel rotation degrees before drinking are more complex than after drinking. The main reason is that the normal driver is cautious and controls the vehicle through continuously adjusting the steering wheel, while the drunk driver is careless, less likely to make gradual adjustment of the steering wheel.

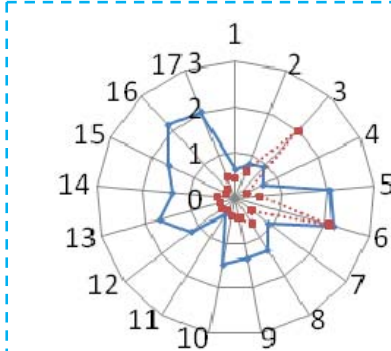

(a)Mean time interval between releasing throttle and wheel angle on the braking

$\longrightarrow$ Normal driving

......... Drunk driving

Fig. 7: Contrasts of time interval and steering parameter

Table 3: The significance Analyses of Characteristic Parameters of Operation Behavior

\begin{tabular}{|c|c|c|c|c|c|}
\hline Parameter & \multicolumn{2}{|c|}{ Mean } & \multicolumn{2}{c|}{ Standard deviation } & P value \\
\hline & Normal & Drunk & Normal & Drunk & \\
\hline Speed of releasing clutch when starting vehicle & 0.211059 & 0.370047 & 0.041513 & 0.088072 & 0 \\
\hline Speed of releasing clutch when shifting gears & 1.882251 & 3.617974 & 0.711086 & 0.670066 & 0 \\
\hline Depth of pedaling throttle & 0.396715 & 0.497892 & 0.072882 & 0.069038 & 0 \\
\hline Speed of pedaling throttle when starting vehicle & 0.117352 & 0.153717 & 0.049946 & 0.063129 & 0.021 \\
\hline Speed of pedaling throttle when accelerating & 0.327790 & 0.661375 & 0.138213 & 0.249599 & 0 \\
\hline Brake times & 3.176470 & 1.705882 & 0.951005 & 0.469668 & 0 \\
\hline Brake depth & 0.327884 & 0.425882 & 0.077438 & 0.050101 & 0 \\
\hline Brake speed & 0.251911 & 0.209752 & 0.072220 & 0.068841 & 0.140 \\
\hline Interval between releasing throttle and braking & 1.339082 & 0.605882 & 0.547990 & 0.540422 & 0.001 \\
\hline S.D of steering wheel rotation degrees & 0.010031 & 0.008874 & 0.001727 & 0.001126 & 0.034 \\
\hline
\end{tabular}

The significance analysis of the above characteristic parameters of driving performance is shown in Table 3.The analysis results show that drinking affects almost all of the operation behaviors to some degree. The changes of the pedal operation are especially significant. All of the impacts have the common characteristics of reducing the vehicle stability and increasing the traffic crash risk.

Based on the above analysis result of impact of alcohol on driving behavior and considering the feasibility of detection of the characteristic parameters, the following parameters were selected: acceleration, deceleration, 
speed of releasing clutch when starting vehicle, speed of releasing clutch when gearing, depth of pedaling throttle, speed of pedaling throttle when accelerating, brake depth. They were comprehensively analyzed to evaluate driving states.

\section{Identifying Method of Drunk Driving}

\subsection{Discrimination Analysis}

In our study, the purpose is to classify the two driving states, normal and drunk driving. Feature selection for classifiers is very important ${ }^{16}$. And feature selection has two main objectives: 1 ) to select a small feature subset and 2) to maintain high classification accurac ${ }^{17}$. To construct the classifier, the seven chosen indicators which were significantly different in two states shown in Table 2 and 3 were selected. Fisher Discriminant Method was used to evaluate driving state and obtain the discriminant function in the study.

Here, $x_{1} \sim X_{7}$ represented the indicator of the characteristic parameters. Their corresponding relations are as follows: $x_{1}$ : Acceleration, $x_{2}$ : Deceleration, $x_{3}$ : Depth of pedaling throttle, $x_{4}$ : Speed of releasing clutch when starting, $x_{5}$ : Speed of releasing clutch when accelerating, $x_{6}$ : Speed of pedaling throttle, $x_{7}$ : Brake depth.

According to the above analysis, it appears that each indicator in two states showed significant differences. And we considered the hypothesis that the covariance matrices were equal at the significance level of 0.05 . Furthermore, each indicator variable obeyed normal distribution at the significance level of 0.05 . So Fisher discriminant method can be used to classify the two states. Driving state was defined as Y, with "0" for normal, " 1 " for drunk. The indicators of the 17 drivers were analyzed by discriminant module in SPSS. The results showed that the discriminant function was statistically significant at the significance level 0.01 . The standardized function is: $y=0.437 x_{1}^{*}+0.447 x_{2}^{*}+0.649 x_{3}^{*}+0.418 x_{4}^{*}+0.451 x_{5}^{*}-0.229 x_{6}^{*}-0.067 x_{7}^{*}$

Where $y$ is the discriminant score, and $x_{i}^{*}(i=1,2, \ldots, 7)$ means the standardized indicator. The coefficient means the weight of each variable, explaining the importance of corresponding variables. The unstandardized function is: $y=-12.236+2.007 x_{1}+0.943 x_{2}+9.422 x_{3}+0.605 x_{4}+6.157 x_{5}-3.900 x_{6}-1.029 x_{7}$
Where $x_{i}(i=1,2, \ldots, 7)$ denotes the original variable of indicators. Discriminant score of each driving state can be calculated through the above function directly. According to the group centroids of discriminant score for two states were $-2.368,2.368$, and because the two groups have the same size, the optimal critical score was defined at 0 . A driver's state discriminant score can be calculated by Equation (2). Then we can classify drivers' state according to the score. A driver is considered to be drunk driving when his/her score exceeds 0 , and normal driving when his/her score is less than 0 .

The 17 drivers' states were classified based on the discriminant function. The accuracy of classifying reached $100 \%$, and the accuracy reached $97.1 \%$ when cross validated, in which each case is classified by the functions derived from all cases other than this case.

\subsection{Analysis and Verification}

The other 7 drivers' data was used to validate the accuracy of identifying function. The 14 group data was calculated according to Equation (2), and the discriminant score was shown as in Fig.8. Because "0" is the optimal critical score, the figure shows that all of the drinking states were classified correctly, and two of the normal states (drivers 2 and 6) were mistakenly classified. So the accuracy of classification reached $85.7 \%$.

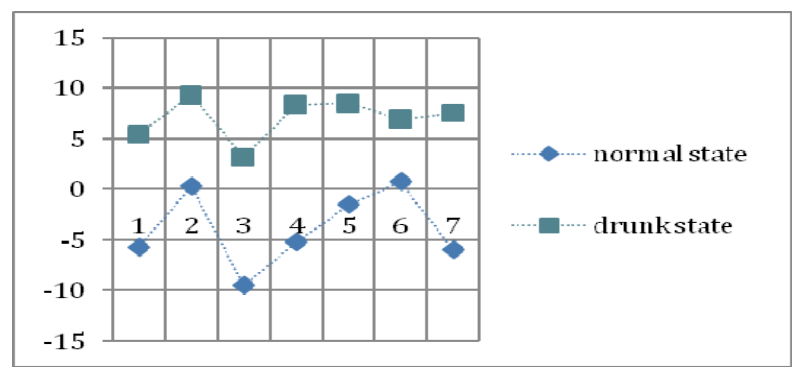

Figure 8: Discriminant Score of the 14 states

The study analyzed the differences between normal driving and drunk driving and developed the discriminant function to classify the two states. The scores for drunk driving were found to be higher than that for normal driving. The score can be considered to be a complex indicator of drunk driving, which represents the effects of alcohol on driver's performance. 


\section{Conclusion}

Alcohol reduces drivers' driving ability and more likely causes traffic crashes since the operating ability of the driver is seriously impaired. According to the analysis of the operating characteristics of drunk driving behaviors, the paper investigated the effect of alcohol on driving behavior and proposed an indicator identifying for drunk driving. The conclusions are as follows:

- Drivers under the influence of alcohol tend to speed and accelerate/decelerate abruptly.

- Drivers under the influence of alcohol are likely to release clutches faster, and push gas pedals faster and deeper when driving on the accelerating segment.

- Drivers under the influence of alcohol tend to push brakes harder and quicker when driving on the decelerating segment.

- Drivers under the influence of alcohol tend to have fewer maneuvers for adjusting vehicle direction on the turning segment.

- When drunk driving, the drivers' reaction ability will go down, and the time interval between releasing throttle and pedaling brake will be shorter.

- The Fisher Discriminant method was used to classify drunk driving performance from normal driving, and the function was developed. The result indicates the higher classification accuracy.

This study focused on the differences of driving behavior in normal driving and drunk driving. The analysis results reveal that there are remarkable differences between the two states and an indicator based on the driver behavior to recognize the drunk driving is developed. Further analysis shows that the accuracy for identifying drunk driving is promising. The identifying method based on driving behavior has shown great significance in practical use. Further research may concentrate on the quantitative relationship between them to obtain an online identifying model of drunk driving.

\section{References}

1. Ravenholt RT. Addition mortality in the United States 1980: tobacco, alcohol and other substances. Population and Development Review. 10(1984) 697-724.

2. Hayakawa H, Fischbeck PS, etc. Traffic accident statistics and risk perceptions in Japan and the United States. Accident Analysis and Prevention, 32(2000) 827-835.
3. Norris FH, Matthens BA, Raid JK. Characterological, situational, and behavioral risk factors for motor vehicle accident: a prospective examination. Accident Analysis and Prevention. 32 (2000) 505-515.

4. Babor T, Caetano R.et al. Alcohol: no ordinary commodity. Research and policy. Oxford University Press. (London,2003)

5. Le Jinghong, Song yongtengye. Effect of a small self-control alcohol dose on skilled driving performance. Psychological Science. 22(1999) 120-122.

6. Amedt JT, Wilde GJ.et al. Simulated driving performance following prolonged wakefulness and alcohol consumption: separate and combined contributions to impairment. $J$ Sleep Res. 9(3) (2000) 233-241.

7. Weiler JM, Bloomfield JR etc. Effects of fexofenadine, diphenhydramine, and alcohol on driving performance: a randomized, placebo-controlled trial in the Iowa driving simulator. Annual of Internal Medicine. 132 (2000) 354-363.

8. Jinghong Yue, Cao Ningning. The effects of alcohol myopia and alcohol hyperopia in are thematic operation-cognition. Psychological Science. 25(3) (2002) 293-295

9. Jinghong Yue, Tengye SONGYONG, et al. Study on the Effect of Self-controlling little Alcohol on Driving Performance. Psychological Science. 2 (1999) 20-123

10. CHEN Cong, ZHUO Xian-yi, SHEN Bao-hua. A Statistics of Alcoholic Driving in Pudong District of Shanghai. Chinese Journal of Forensic Sciences. 1 (2010) 37-39.

11. YIN Kai, LI Ming-qiang etc. The relativity of alcohol and traffic accidents. Chin JDis Control Prev. 11(4) (2007) 392-394.

12. Soyka M, Aichmuller C. et al. Effects of acamprosate on psychomotor performance and driving ability in abstinent alcoholics. Pharmacopsychiatry, 31(1998) 232- 235.

13. WANG Gen-sheng, CHEN Zi-qiang, et al. Effects of driving on human nervous behavior function. Chinese Journal of Preventive Medicine. 33(6) (1999) 380-381.

14. LI Chun-ming, LIU Hao-xue. Research of visual behavior of drunk driver. Automative Researching. 15(1) (1996) 21-24.

15. Amedt JT, Wilde GJ, etc. How do prolonged wakefulness and alcohol compare in the decrements they produce on a simulated driving task. Accident Analysis and Prevention, 33 (2001)337-344.

16. Z. Wei and D. Miao, N-grams based feature selection and text representation for Chinese Text Classification. International Journal of Computational Intelligence Systems.2 (4) (2009) 365 - 374.

17. Liming Yang, Laisheng Wang, et al. Simultaneous feature selection and classification via Minimax Probability Machine. International Journal of Computational Intelligence Systems.3 (6) (2010) 754 - 760. 